\title{
IV. Die Reichstagswahlen vom Mai und Dezember 1924: Debakel der Großen Koalition
}

Bei den Reichstagswahlen vom 4. Mai 1924 erzielten die Parteien der Großen Koalition ein insgesamt miserables Ergebnis 1 . Zusammengenommen kamen sie nur noch auf knapp 50\% der Stimmen, und mit 238 Mandaten lagen sie nur minimal über der arithmetischen Mehrheit von 236 Abgeordneten². Besonders schlecht schnitt die wiedervereinigte SPD ab, die mit 20,5\% sogar noch hinter dem Resultat der MSPD aus dem Jahr 1920 (21,7\%) zurückblieb3. Rechnet man die 17,9\% ein, die 1920 von der USPD erreicht worden waren, dann ergibt dies einen Gesamtverlust von $19,1 \%$, d.h. das sozialdemokratische Wählerreservoir wurde innerhalb von vier Jahren fast halbiert. Massive Verluste mußte auch die DVP einstecken. Von 13,9\% bei den Wahlen 1920 sank die Partei Stresemanns auf 9,2\% ab. Ähnlich erging es auf niedrigerem Niveau der DDP, die von 8,3 auf $5,7 \%$ rutschte. Relativ stabil blieb mit $13,4 \%(-0,2 \%)$ lediglich die Zentrumspartei. Die großen Wahlsieger waren die DNVP mit 19,5\% $(+4,4)$ bzw. $21,5 \%$ zuzüglich des DNVPnahen Landbundes, die KPD mit 12,6\% (+10,5) sowie die diversen völkischnationalsozialistischen Wahllisten, die aus dem Stand reichsweit auf 6,5\% kamen. Einen erheblichen Stimmenzuwachs konnten auch mehrere Splitterparteien verbuchen, die insgesamt auf etwa 9,5\% kamen.

Stärkste Reichstagsfraktion wurde nun die mit dem Landbund verbundene DNVP (95+10 Abgeordnete), gefolgt von der stark geschrumpften SPD (100 statt zuletzt 171), der Zentrumsfraktion (65), der KPD (62), DVP (45), den Völkischen und Nationalsozialisten (32), der DDP (28) und der BVP (16). Die sonstigen kleinen Gruppen erreichten zusammen eine Mandatszahl von 19. Die Möglichkeiten der parlamentarischen Mehrheitsbildung, für die 237 Abgeordnete notwendig waren, wurden mit diesem Ergebnis noch weiter beschränkt. Das Modell der Weimarer Koalition kam rechnerisch nur noch auf 193 Mandate. Eine Große Koalition wäre nur unter Hinzunahme der BVP wirklich mehrheitsfähig gewesen (238+16). Ein Bürgerblock unter Einbeziehung der DNVP4 lag zwar etwas deutlicher über der theoretischen Mehrheitsmarke, aber auch hier hätte erst eine Beteiligung der BVP für eine klare Mehrheit gesorgt (243+16). Extrem schwierig war die Situation für eine Minderheitsregierung der bürgerlichen Mitte, die nur noch auf 138 Sitze kam. Selbst eine Tolerierung durch SPD oder DNVP erbrachte hier noch keine tragfähige Mehrheit.

1 Vgl. zum Ergebnis Anhang, Tab. 4.1 und 4.2. Die Reichstagswahlen fanden nun doch eine Woche vor den französischen Kammerwahlen statt. Zur Diskussion um den Wahltermin vgl. oben S. 334, Anm. 584.

2 Die BVP ist hier jeweils ausgenommen.

3 Winkler, Weimar, S. 261, spricht hier zu Recht davon, daß dies „einer Katastrophe nahe“ kam. Allgemein zu dem Ergebnis ebd., S. 261 f. Detaillierte Analyse des sozialdemokratischen und kommunistischen Ergebnisses in ders., Schein, S. 177-188. Vgl. zur Analyse des Wahlverhaltens und zu Mutmaßungen über Wählerwanderungen auch Maier, Recasting Bourgeois Europe, S. 450-455; Falter, Hitlers Wähler, S. 26-28; Lau, Wahlkämpfe der Weimarer Republik, S. 292 f.; Jones, German Liberalism, S. 220-222.

4 Zuzüglich Landbund. 
Die Erklärungen, die dieses Ergebnis in der Literatur findet, bleiben oft merkwürdig unbestimmt. Insgesamt dominiert die Bewertung als "Inflationswahl“5, was zwar nicht falsch, aber doch etwas irreführend ist. Wäre das Resultat vom 4. Mai hauptsächlich eine unmittelbare Folge der Hyperinflation und der mit ihr verbundenen sozialen und politischen Erschütterungen gewesen, dann müßte die Vermutung Winklers zutreffen, bei einer Wahl im Sommer oder Herbst 1923 „hätten die radikalen Kräfte von rechts und links mit Sicherheit noch mehr Zulauf gehabt" 6 . Mangels zeitgenössischer demoskopischer Erhebungen läßt sich diese These nur schwer verifizieren oder auch falsifizieren. Es gibt aber doch eine Reihe von Indizien, die ihr eher widersprechen. So erbrachten die drei Landtagswahlen im Sommer und Herbst 1923 - Oldenburg (10.6.), Mecklenburg-Strelitz (8. 7.) und Bremen (18. 11.) - tendenziell ähnliche Ergebnisse wie die Reichstagswahl vom Mai 1924. Alles in allem lagen die Verluste von SPD und bürgerlicher Mitte jedoch nicht über jenen vom Mai 1924. Dasselbe gilt für die drei Landtagswahlen vom Februar 1924 in Lübeck (10.2.), Thüringen und Mecklenburg-Schwerin (jeweils 17. 2.).

Geht man davon aus, daß ein Reichstagswahltermin im Herbst 1923 vermutlich kein schlechteres Ergebnis für die systemtragenden Parteien erbracht hätte und hält man gleichzeitig die Annahme Winklers für plausibel, daß ein gewisses Abflauen der politischen Erregung seit Herbst 1923 den gemäßigten Kräften zugute gekommen ist ${ }^{8}$, dann muß es auch einen umgekehrten Trend gegeben haben, der zahlreiche Wähler seit Herbst 1923 zu einer Wahlentscheidung für die extremen Kräfte und vor allem für die DNVP bewogen hat. Hier liegt die Vermutung nahe, daß es die konkreten Stabilisierungsmaßnahmen waren, die mit wahlentscheidend wurden'. Besonders die von der DNVP sofort demagogisch im Wahlkampf aufgegriffene Frage der Schuldenaufwertung dürfte eine Rolle gespielt haben ${ }^{10}$.

Ein anderer Erklärungsansatz zielt auf die zuletzt immer mehr in den Mittelpunkt des Wahlkampfes ${ }^{11}$ gerückte Diskussion um den Dawes-Plan. Die Regierung Marx hatte das kurz zuvor publizierte Gutachten der eingesetzten Sachverständigenkommission am 14. April in einer Note an die Reparationskommission

5 Vgl. z.B. Falter, Hitlers Wähler, S. 26. Allgemein zur Bewertung in der Forschung vgl. auch Lau, Wahlkämpfe, S. 254.

6 Winkler, Weimar, S. 262.

7 Zahlen zu den Landtagswahlen nach Falter, Wahlen, S. 93, 97-99, 111.

8 Hierauf deutet auch, daß alle Parteien der Großen Koalition im Wahlkampf um das Hauptverdienst bei der Währungsstabilisierung stritten und daß auch die Regierung in diesem Sinne propagandistisch tätig war. Vgl. auch einen Aufsatz von Hugo Heimann, Vorsitzender des Haushaltsausschusses, in Vo, 11. 4. 1924, 2. Beilage, S. 1f., „Inflation und bürgerliche Parteien“. „Die Schuld am Zusammenbruch der Währung, das Verdienst an ihrer vorläufigen Stabilisierung spielen im Wahlkampf eine besondere Rolle, und jede Partei nimmt für sich in Anspruch, die Währung stabilisiert und damit Deutschland vor dem Chaos gerettet zu haben." Als Beispiel für Wahlpropaganda vgl. z. B. Luther, Feste Mark - Solide Wirtschaft.

9 Vgl. auch Jones, German Liberalism, S. 220. Diese These entspricht der Annahme in der neueren Forschung, daß eine Kompromittierung des parlamentarischen Systems weniger durch die Inflation als durch „Form und Folgen der Mark-Stabilisierung" bewirkt wurde. So zusammenfassend Schneider, Deutsche Gesellschaft in Krieg und Währungskrise 1914-1924, S. 317.

10 Vgl. auch Jung, Direkte Demokratie, S. 17.

"Vgl. hierzu Lau, Wahlkämpfe, S. 254-295. Die von Bendikat/Lehnert, "Schwarzweißrot gegen Schwarzrotgold" vorgetragene These ist zu pauschal. 
akzeptiert ${ }^{12}$. Trotz der heftigen Polemik, die die DNVP sofort gegen das „zweite Versailles" entfachte, scheint keineswegs eindeutig, welchen Einfluß diese Frage auf das Wahlergebnis gewonnen hat ${ }^{13}$. So wurde die außenpolitische Thematik von der Regierung und den regierungstragenden Parteien teilweise sogar bewußt in den Vordergrund gestellt, um „von den unpopulären ,Opfern' der Spar- und Sanierungspolitik im Innern abzulenken " 14 .

Kaum thematisiert wurden bisher in der Literatur die Zusammenhänge zwischen dem Wahlergebnis und den vorhergehenden parlamentarischen Entwicklungen ${ }^{15}$. Anknüpfend an unsere Krisenanalyse seien daher im folgenden einige thesenhafte Überlegungen angestellt.

Im Hinblick auf die SPD läßt sich das Ergebnis auch als Quittung für die Tolerierung von Mitte-rechts-Regierungen (Cuno und Marx) bzw. für die Beteiligung an einer Großen Koalition deuten. Die jetzt erfolgte massive Wählerwanderung zu den Kommunisten war der wiedervereinigten Sozialdemokratie von dem liberalen Publizisten Hellmut von Gerlach bereits anläßlich der Koalitionsdiskussion im Oktober 1922 prophezeit worden ${ }^{16}$. Wie das gute Wahlergebnis der SPD im November 1922 in Sachsen nahelegt ${ }^{17}$, war der vor allem von einstigen USPDWählern vollzogene Wechsel zur KPD keineswegs ein zwangsläufiger Prozeß, sondern hatte offenbar wesentlich mit den Vorgängen seit Ende $1922 \mathrm{zu}$ tun. Generell ist hier zunächst die Verbitterung innerhalb der Arbeiterschaft nach zahlreichen verlorenen Arbeitskämpfen und dem inflationsbedingten Bankrott der Gewerkschaften als radikalisierender Faktor zu berücksichtigen. Ein politisches Grundproblem lag zweifellos darin, daß es der SPD 1923 nicht gelang, ihre Regierungsunterstützung bzw. -beteiligung auch in entsprechenden Einfluß auf das Regierungshandeln umzumünzen. Die SPD war, obwohl sie die weitaus stärkste Reichstagsfraktion stellte, in ihrer formellen und informellen Kooperation mit der bürgerlichen Mitte, politisch weitgehend „kaltgestellt“18. Einzelne regionalgeschichtliche Untersuchungen zeigen, daß an der enttäuschten Basis eine wachsende Resignation herrschte ${ }^{19}$. Für besondere Verärgerung hat neben dem mona-

12 Vgl. Krüger, Außenpolitik, S. 239.

13 Liebe, Deutschnationale Volkspartei, S. 77 f., stellt diese Frage unter Ausblendung anderer Themen in den Mittelpunkt.

14 Lau, Wahlkämpfe, S. 294.

15 Winkler, Schein, S. 182-187, gibt zwar eine detaillierte Analyse des sozialdemokratischen und kommunistischen Wahlergebnisses, bleibt aber bei der Erklärung blaß. Der Zusammenhang mit der Politik der SPD wird - in sehr allgemeiner Form - noch am ehesten bei Maier, Recasting Bourgeois Europe, S. 454, und bei Mommsen, Verspielte Freiheit, S. 196 f., betont.

16 Gerlach warnte dabei nachdrücklich vor der Großen Koalition: „Die Situation der Sozialdemokratie ist keineswegs leicht. Sie mit ihren 180 Abgeordneten scheint ein Riese gegenüber dem Zwerg Kommunismus mit seinen kümmerlichen 11 Vertretern. Aber die Not der Zeit könnte dem Zwerge Riesenkraft verleihen, wenn der Goliath nicht mit der Länge seiner Gestalt Stärke des Rückgrats zu vereinigen wüßte. [...] Aber in der Regierung sitzen und kaltgestellt sein, alles verantworten zu müssen und nichts zu sagen zu haben - das geht nicht länger an. Das diskreditiert die Partei bis auf die Knochen. Das bereitet den Kommunisten den Weg." WaM, 30. 10. 1922, S. 1, „Wirtschaft, Herr Wirth!“

17 Vgl. oben S. 151.

18 Vgl. die in Anm. 16 zitierte Prognose Gerlachs.

19 Vgl. z. B. Rother, Die Sozialdemokratie im Land Braunschweig 1918 bis 1933, S. 177. Dramatische Einbrüche beim Wahlergebnis gab es demnach v.a. in ehemaligen USPD-Hochburgen. Zur resignativen Stimmung vgl. auch Müller, Zwischen Wahlkampf und Politik, S. $504 \mathrm{f}$. 
telangen militärischen Ausnahmezustand wohl die Zustimmung zu den Ermächtigungsgesetzen gesorgt. Dies ist nicht allein an den kontroversen Diskussionen um die Verabschiedung ablesbar, sondern auch an der Kandidatenaufstellung für die Wahlen vom Mai 1924. So berichtete Hermann Müller auf dem Berliner SPDParteitag vom Juni 1924, daß ,in verschiedenen Wahlkreisen [...] in Bausch und Bogen diejenigen Abgeordneten abgesägt worden [seien], die sich für die Ermächtigungsgesetze ausgesprochen haben " 20 . Wie ein Blick auf die Zusammensetzung der sozialdemokratischen Fraktion in der zweiten Wahlperiode zeigt, hatten es vor allem ehemalige USPD-Abgeordnete, die für die Ermächtigungen votiert hatten, sehr schwer, wieder in den Reichstag einzuziehen ${ }^{21}$.

Inwieweit die von der SPD seit dem Ausscheiden aus der Regierung Stresemann verfolgte Politik einer eher symbolischen Opposition und eines ansatzweisen Aufzeigens parlamentarischer Alternativen Einfluß auf das Wahlergebnis gewonnen hat, ist schwer zu sagen. Die Annahme liegt aber nahe, daß es ohne Regierungsaustritt, ohne Antrag auf ein Mißtrauensvotum gegen Stresemann und ohne die zuletzt inszenierten Bemühungen um Veränderungen am Verordnungswerk der Regierung Marx möglicherweise noch schlechter ausgefallen wäre.

Stark geschwächt wurde auch die andere Flügelpartei der Großen Koalition. Die herben Verluste der DVP, die sich vermutlich vor allem aus einer Abwanderung von Wählern an die DNVP ergaben, lassen sich zunächst ganz unmittelbar mit den durch die Stabilisierungsverordnungen getroffenen Maßnahmen, insbesondere mit dem eingeleiteten Personalabbau im Staatsdienst und mit einem für zu gering gehaltenen Satz bei der Schuldenaufwertung erklären. Sie resultierten aber wohl auch daraus, daß die DVP mit der SPD in der Regierung kooperiert hatte und daß es darüber zu heftigen parteiinternen Konflikten gekommen war. Die Spannung zwischen Stresemann und dem rechten Flügel seiner Partei war zudem kurz vor den Wahlen mit der Gründung und dem kurz darauf folgenden Parteiausschluß der Nationalliberalen Vereinigung eskaliert. Die Abgeordneten Vögler und Quaatz, neben dem am 10. April überraschend verstorbenen Stinnes die führenden Vertreter dieser Tendenz, hatten darauf mit einem Wahlaufruf zugunsten der DNVP reagiert ${ }^{22}$. Jetzt, kurz vor den Reichstagswahlen, wurde schließlich der Trennungsstrich zu jener intransigenten schwerindustriellen Gruppe innerhalb der DVP gezogen, die im Herbst 1923 nicht unwesentlich zum Scheitern der Großen Koalition beigetragen hatte.

20 Bericht der Reichstagsfraktion, in: Sozialdemokratischer Parteitag 1924, S. 80-92, hier S. 90. Auf dem Parteitag wurde teilweise scharfe Kritik an den Ermächtigungsgesetzen geübt.

2125 der 33 SPD-Abgeordneten aus den Reihen der ehemaligen USPD, die beim Votum vom 8. 12. 1923 mit "Ja" gestimmt hatten, saßen nicht mehr im zweiten Reichstag; das sind etwa $76 \%$. Insgesamt zogen etwa $54 \%$ der Abgeordneten, die der SPD-Fraktion am Ende der ersten Wahlperiode angehört hatten, nicht mehr in den zweiten Reichstag ein. Berechnung nach Liste der namentlichen Abstimmung, Verh. RT 361, S. 12359-12386, und nach Reichstagshandbuch, II. Wahlperiode 1924.

22 Vgl. Sitzung des Zentralvorstands der DVP am 28. 3. 1924 in Hannover, Protokoll in Nationalliberalismus, Nr. 55, S. 490 f., sowie ebd. Anm. 4; Jones, German Liberalism, S. 214-216; Richter, Deutsche Volkspartei, S. 303-322. 
Die Unzufriedenheit in der Beamtenschaft über den Stellenabbau per Verordnungsregime schadete sicher auch der DDP23. Darüber hinaus rief aber, wie bereits erwähnt, die ausufernde Verordnungspraxis an der linksliberalen Basis auch grundsätzliche Kritik hervor, die von der Sorge um die freiheitliche und parlamentarische Ordnung getragen war $^{24}$.

Der große Wahlsieg der DNVP wäre vermutlich ausgeblieben, wenn es im Herbst und Winter 1923 gelungen wäre, die Deutschnationalen in irgendeiner Form in das parlamentarische Regierungslager einzubinden. Statt dessen hatte die Partei im Wahlkampf die Themen Dawes-Plan und Schuldenaufwertung zu einer hemmungslosen Fundamentalopposition nutzen können ${ }^{25}$. Allerdings wäre bei einer deutschnationalen Mitverantwortlichkeit für die Regierungspolitik möglicherweise der völkisch-nationalsozialistische Erfolg über den erreichten 6,5\% gelegen.

Die neuen Mehrheitsverhältnisse im Reichstag bildeten für die jetzt anstehende Normalisierung und Wiederbelebung des parlamentarischen Systems eine sehr brüchige Grundlage. Ein symbolkräftiges Indiz für die schwierige Situation waren die von der KPD veranstalteten tumultartigen Szenen beim Zusammentritt des Reichstags am 27. Mai 192426. Von den gescheiterten Koalitionsverhandlungen der Regierungsparteien mit der DNVP und der Demission des Kabinetts Marx I über die Bildung des Minderheitskabinetts Marx II und die Verhandlungen um eine Regierungserweiterung ${ }^{27}$ bis zur erneuten Auflösung des Reichstags am 20. Oktober 1924 spannte sich ein Bogen des Mißerfolgs in der regierungstragenden Funktion. Dies wiederum lähmte insgesamt auch die legislative Tätigkeit. Positiv ist allerdings hervorzuheben, daß am 29. August eine breite Reichstagsmehrheit den Dawes-Plan annahm. Dank eines gespaltenen Abstimmungsverhaltens der DNVP-Fraktion gelang es in der Frage der notwendig werdenden Umgestaltung der Reichsbahn sogar, die hierfür erforderliche verfassungsändernde Zweidrittelmehrheit zu erreichen. Im Zeichen der positiven Währungs- und Wirtschaftsentwicklung löste sich die Weimarer Republik jetzt auch wieder von den Instrumenten des Verordnungsregimes. Von einem neuen Ermächtigungsgesetz war keine Rede mehr, und der legislative Einsatz von Artikel 48 ging im Laufe des Jahres 1924 deutlich zurück, um dann über Jahre hinweg ganz eingestellt zu werden ${ }^{28}$.

23 Vgl. auch Analyse durch Erkelenz in der Vorstandssitzung der DDP vom 21. 5. 1924; BA Koblenz, R 45 III, Nr. 19, B1. 76.

${ }_{24}$ Vgl. oben S. 330. - Genauere Untersuchungen über die Rezeption vor allem des zweiten großen Ermächtigungsgesetzes innerhalb der DDP erscheinen in diesem Zusammenhang sinnvoll.

25 Zu den Ursachen des DNVP-Wahlerfolgs vgl. auch Pyta, Dorfgemeinschaft und Parteipolitik, S. 295, der die Zurückweisung ,jeglicher[r] politische[r] Verantwortung für den unpopulären Kurs in der Finanz-und Außenwirtschaftspolitik" betont.

26 Erstmals in der Weimarer Republik wurde das Anfangsritual des Reichstags massiv gestört. Die erstarkte kommunistische Fraktion lärmte u.a. mit Schlüsseln und Kindertrompeten. Verh. RT 381, S. 1-6. Vgl. hierzu Mergel, Parlamentarische Kultur, S. 141 f.

27 Im Sinne einer Rechtserweiterung oder einer Großen Koalition oder gar einer extrem großen Koalition der "Volksgemeinschaft" von der SPD bis zur DNVP.

28 Vgl. Übersicht von Poetzsch (ab 2: Poetzsch-Heffter), Staatsleben 1, S. 147, und 2, S. 99. Immerhin waren es aber auch 1924 noch 13 legislative Verordnungen (ohne Aufhebungsverordnungen). Die vorläufig letzte legislative Verordnung nach Art. 48 wurde am 29.1.1925 erlassen (Aufnahme von Auslandskrediten durch Gemeinden und Verbände). Knapp zur Praxis 1924 Kurz, Demokratische Diktatur?, S. 159. Zur vergeblichen Forderung Strescmanns im November 1924, eine Erhöhung der Zolltarife mit Hilfe von Art. 48 durchzusetzen, vgl. BA Berlin, R 3001, Nr. 6631, Bl. 252-256. 
Bei den Neuwahlen zum Reichstag am 7. Dezember konnten die systemloyalen Parteien ihr katastrophales Ergebnis vom 4. Mai ein wenig verbessern. DDP, DVP und Zentrum erzielten minimale Gewinne. Die SPD machte von ihren erdrutschartigen Verlusten 5,5\% wieder gut und erhöhte ihren Stimmenanteil auf $26 \%$, was freilich immer noch 13,6\% unter dem addierten Ergebnis von MSPD und USPD aus dem Jahre 1920 blieb. Die DNVP legte nochmals leicht auf jetzt 20,5\% zu, während die extremen Kräfte von KPD und NSDAP mit Verlusten von jeweils etwa 3,5\% wieder deutlich zurückgeworfen wurden. Die verschiedenen Kleinstparteien lagen in der Summe mit 7,8\% weiterhin relativ hoch.

Für die Möglichkeiten der Regierungsbildung bedeutete dieses Ergebnis nur eine partielle Verbesserung. Das Modell der Großen Koalition wurde jetzt - neben jenem der bürgerlichen Rechtskoalition - wieder mehrheitsfähig, die Weimarer Koalition hingegen lag deutlich unter der neuen Mehrheitsmarke von 247 Mandaten. Die von den Stärkeverhältnissen im Reichstag her gebildete Ausgangsbasis für die weitere Entwicklung des parlamentarischen Systems blieb ungünstig - ungünstiger, als sie es nach den Reichstagswahlen von 1920 gewesen war. 